\title{
Ultra-Fine $\mathrm{WC}_{1-\mathrm{x}}$ Nanocrystals: An Efficient Cocatalyst for the Significant Enhancement of Photocatalytic Hydrogen Evolution on $\mathrm{g}-\mathrm{C}_{3} \mathrm{~N}_{4}$
}

Rui Tong, ${ }^{\mathrm{a}}$ Zhi Sun, ${ }^{\mathrm{b}}$ Xina Wang, ${ }^{\mathrm{c}}$ Shuangpeng Wang, ${ }^{\mathrm{a}, \mathrm{d} *}$ and Hui Pan ${ }^{\mathrm{a}, \mathrm{d} *}$ aJoint Key Laboratory of the Ministry of Education, Institute of Applied Physics and Materials Engineering, University of Macau, Macau, China.

${ }^{b}$ State Key Discipline Laboratory of Wide-Bandgap Semiconductor Technologies, School of Microelectronics, Xidian University, Xi'an 710071, People's Republic of China.

${ }^{c}$ Hubei Key Laboratory of Ferro \& piezoelectric Materials and Devices, Faculty of Physics and Electronic Science, Hubei University, Wuhan 430062, People's Republic of China.

${ }^{d}$ Department of Physics and Chemistry, Faculty of Science and Technology, University of Macau, Macau, China.

*E-mail: spwang@um.edu.mo (S.P.Wang); huipan@um.edu.mo (H.Pan); Fax: +853 88222426; Tel: +85388224427 ;

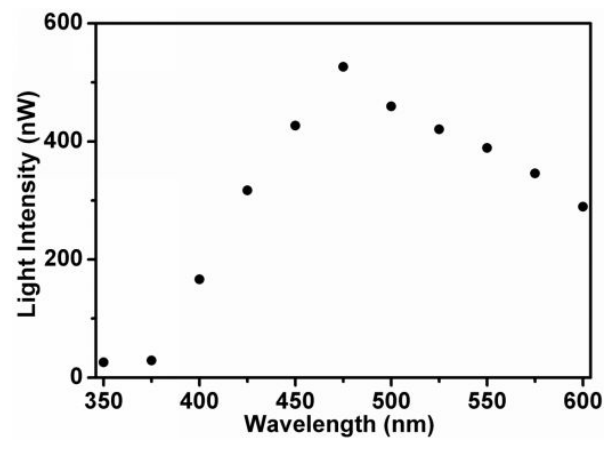

Figure S1. The light intensity distribution of monochromatic wave in IPCE measurement.
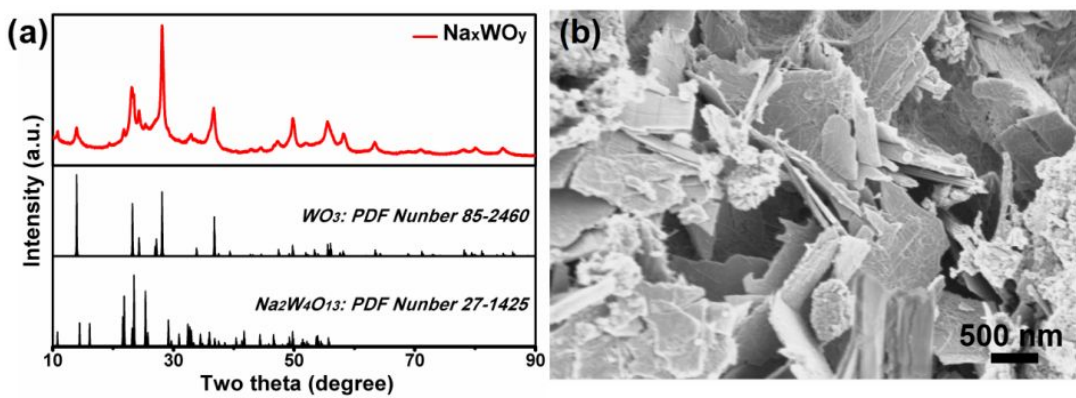

Figure S2. XRD pattern (a) and SEM image (b) of $2 \mathrm{D} \mathrm{Na}_{\mathrm{x}} \mathrm{WO}_{\mathrm{y}}$. 


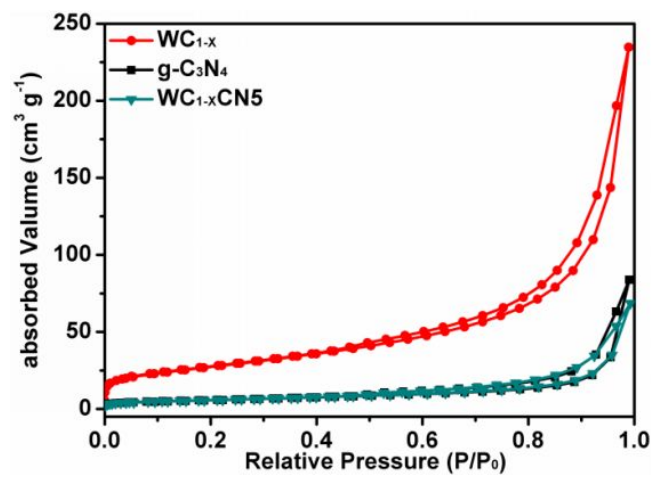

Figure S3. Nitrogen adsorption/desorption isotherms of the $\mathrm{WC}_{1-\mathrm{X}}, \mathrm{g}-\mathrm{C}_{3} \mathrm{~N}_{4}$ and $\mathrm{WC}_{1-\mathrm{X}} \mathrm{CN} 5$.

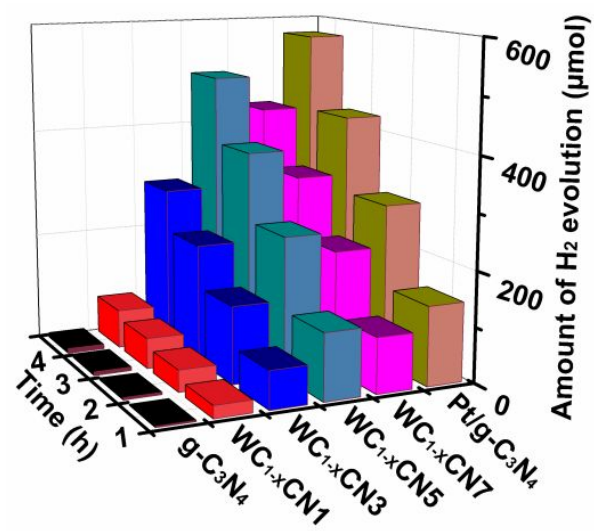

Figure S4. Time-dependent photocatalytic $\mathrm{H}_{2}$ evolution of $\mathrm{g}-\mathrm{C}_{3} \mathrm{~N}_{4}, \mathrm{WC}_{1-\mathrm{x}} \mathrm{CN} 1, \mathrm{WC}_{1-\mathrm{x}} \mathrm{CN} 3, \mathrm{WC}_{1-\mathrm{x}} \mathrm{CN} 5$, $\mathrm{WC}_{1-\mathrm{X}} \mathrm{CN} 7$ and $\mathrm{Pt} / \mathrm{g}-\mathrm{C}_{3} \mathrm{~N}_{4}$.
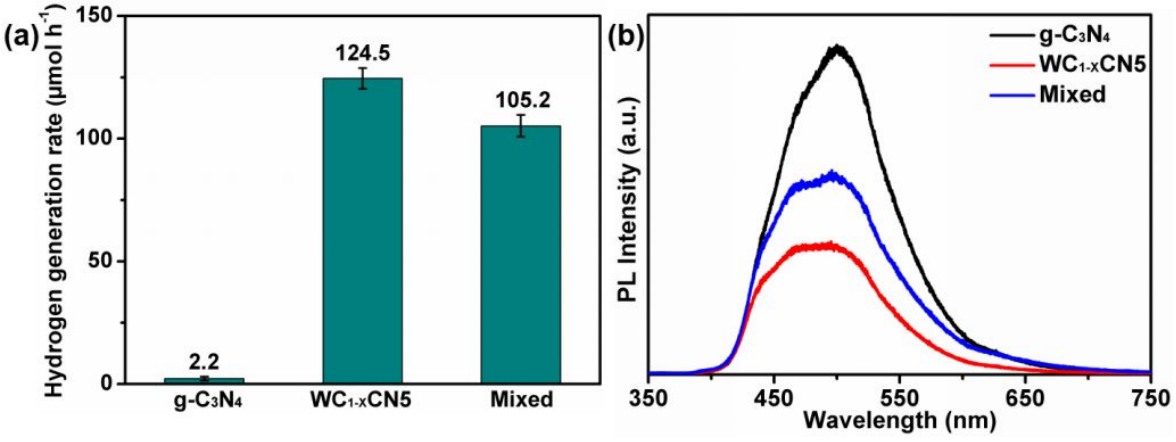

Figure S5. The comparision of the average $\mathrm{H}_{2}$ generation rates (a) and PL spectra (b) of $\mathrm{g}_{-} \mathrm{C}_{3} \mathrm{~N}_{4}, \mathrm{WC}_{1-\mathrm{X}} \mathrm{CN} 5$ and the mixed sample. The error bars in (a) are standard deviation of three tests $(n=3)$. 


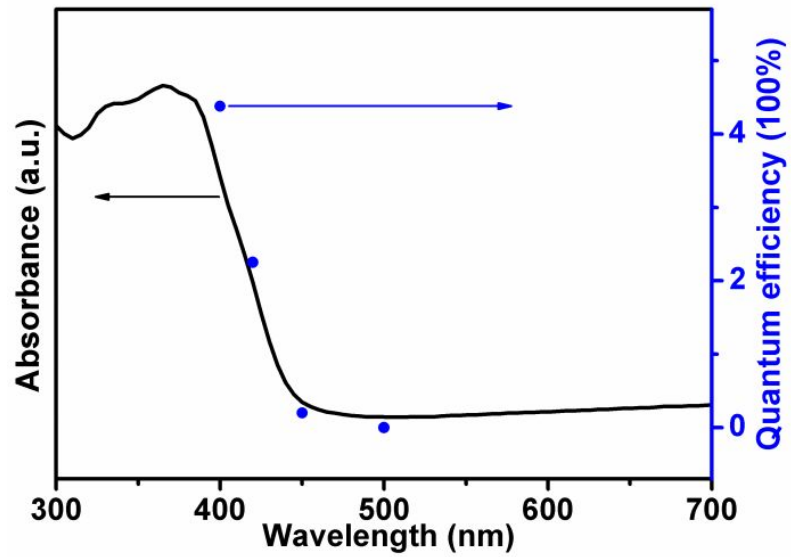

Figure S6. UV-VIS absorption spectrum and action spectrum of $\mathrm{WC}_{1-\mathrm{X}} \mathrm{CN} 5$.

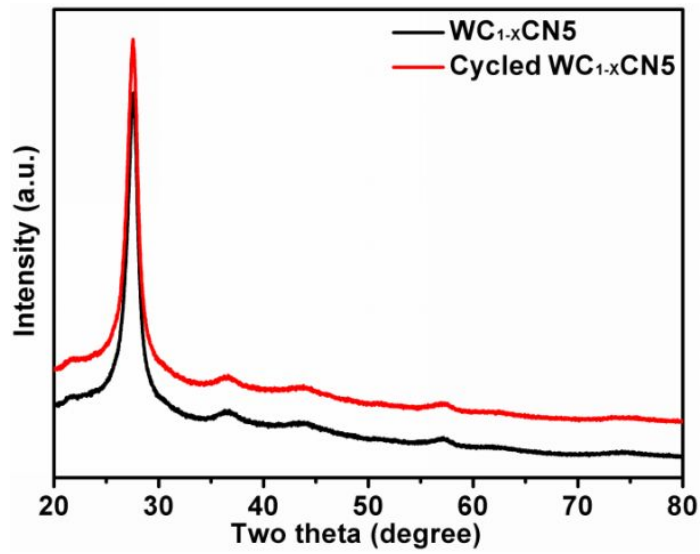

Figure $\mathbf{S} 7$. The XRD patterns of as-prepared $\mathrm{WC}_{1-\mathrm{X}} \mathrm{CN} 5$ and cycled $\mathrm{WC}_{1-\mathrm{X}} \mathrm{CN} 5$.

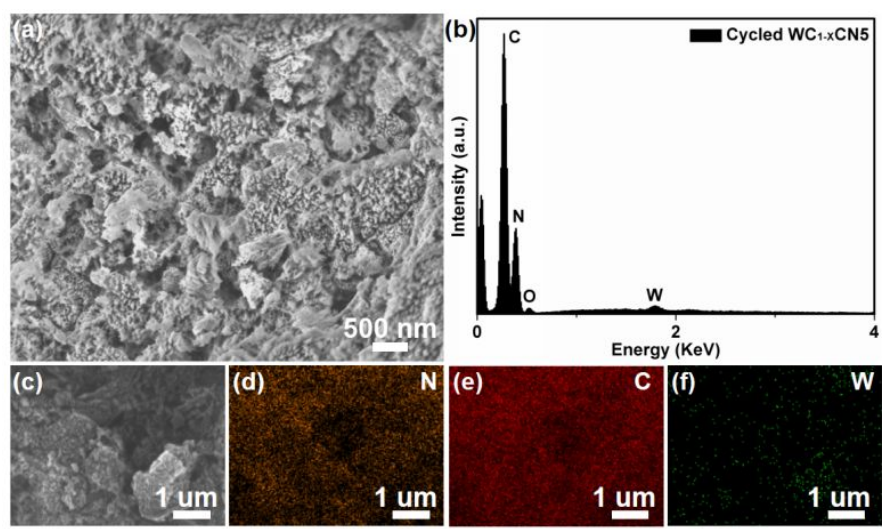

Figure S8. SEM image (a), EDS spectrum (b) and EDS mappings (c-f) of the cycled $\mathrm{WC}_{1-\mathrm{X}} \mathrm{CN} 5$. 

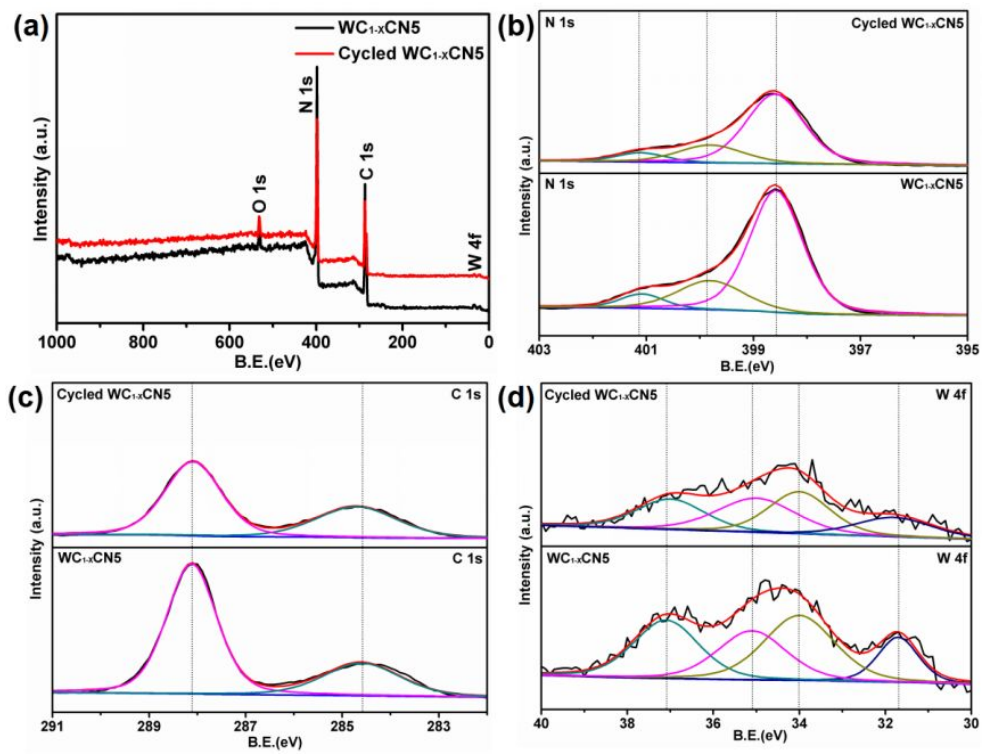

Figure S9. XPS survey spectra (a) and high-resolution XPS spectra of the N 1s (b), C 1s (c) and W 4f (d) of $\mathrm{WC}_{1-\mathrm{X}} \mathrm{CN} 5$ and cycled $\mathrm{WC}_{1-\mathrm{X}} \mathrm{CN} 5$.

Table S1. Comparison of the photocatalytic $\mathrm{H}_{2}$ evolution activity of the $\mathrm{g}-\mathrm{C}_{3} \mathrm{~N}_{4}$-based systems decorated with different cocatalysts.

\begin{tabular}{|c|c|c|c|c|c|}
\hline $\begin{array}{l}\text { Catalysts } \\
\text { (mass) }\end{array}$ & $\begin{array}{c}\text { Cocatalysts } \\
\text { (Loading) }\end{array}$ & Light power & $\begin{array}{c}\text { Activity } \\
\left(\mu \mathrm{mol} \mathrm{h}^{-1} \mathrm{~g}^{-1}\right)\end{array}$ & $\begin{array}{c}\text { AQE } \\
(100 \%)\end{array}$ & Ref. \\
\hline $\begin{array}{c}\mathrm{VS}_{2} / \mathrm{g}-\mathrm{C}_{3} \mathrm{~N}_{4} \\
(50 \mathrm{mg})\end{array}$ & $\begin{array}{c}\mathrm{VS}_{2} \\
(2.8 \mathrm{wt} \%)\end{array}$ & $\begin{array}{c}\text { solar simulator } \\
\text { AM } 1.5\end{array}$ & 1747 & $\begin{array}{c}5.5 \% \\
(420 \mathrm{~nm})\end{array}$ & (1) \\
\hline $\begin{array}{c}\mathrm{Ti}_{2} \mathrm{C} / \mathrm{g}-\mathrm{C}_{3} \mathrm{~N}_{4} \\
(50 \mathrm{mg})\end{array}$ & $\begin{array}{c}\mathrm{Ti}_{2} \mathrm{C} \\
(0.4 \mathrm{wt} \%)\end{array}$ & $\begin{array}{c}\text { solar simulator } \\
\text { AM } 1.5\end{array}$ & 950 & $\begin{array}{c}4.3 \% \\
(420 \mathrm{~nm})\end{array}$ & (2) \\
\hline $\begin{array}{c}\mathrm{WS}_{2} / \mathrm{g}-\mathrm{C}_{3} \mathrm{~N}_{4} \\
(50 \mathrm{mg})\end{array}$ & $\begin{array}{c}\mathrm{WS}_{2} \\
(0.01 \mathrm{wt} \%)\end{array}$ & $\begin{array}{c}300 \mathrm{~W} \text { Xe lamp } \\
(\lambda>420 \mathrm{~nm})\end{array}$ & 101 & --- & (3) \\
\hline $\begin{array}{c}\mathrm{WC} / \mathrm{g}-\mathrm{C}_{3} \mathrm{~N}_{4} \\
(50 \mathrm{mg})\end{array}$ & $\begin{array}{c}\mathrm{WC} \\
(15 \mathrm{wt} \%)\end{array}$ & $\begin{array}{c}300 \mathrm{~W} \text { Xe lamp } \\
(\lambda>420 \mathrm{~nm})\end{array}$ & 146.1 & --- & (4) \\
\hline $\begin{array}{c}\mathrm{Mo}_{2} \mathrm{C} @ \mathrm{C} / \mathrm{g}-\mathrm{C}_{3} \mathrm{~N}_{4} \\
(10 \mathrm{mg})\end{array}$ & $\begin{array}{l}\mathrm{Mo}_{2} \mathrm{C} @ \mathrm{C} \\
(15 \mathrm{wt} \%)\end{array}$ & $\begin{array}{c}300 \mathrm{~W} \text { Xe lamp } \\
(\lambda>400 \mathrm{~nm})\end{array}$ & 2269.47 & $\begin{array}{c}9.07 \\
(405 \mathrm{~nm})\end{array}$ & (5) \\
\hline $\begin{array}{c}\mathrm{Ni}_{3} \mathrm{C} / \mathrm{g}-\mathrm{C}_{3} \mathrm{~N}_{4} \\
(50 \mathrm{mg})\end{array}$ & $\begin{array}{c}\mathrm{Ni}_{3} \mathrm{C} \\
(15 \mathrm{wt} \%)\end{array}$ & $\begin{array}{c}350 \mathrm{~W} \text { Xe lamp } \\
(\lambda>400 \mathrm{~nm})\end{array}$ & 303.6 & $\begin{array}{c}0.4 \% \\
(420 \mathrm{~nm})\end{array}$ & (6) \\
\hline $\begin{array}{c}\mathrm{Mo}-\mathrm{Mo}_{2} \mathrm{C} / \mathrm{g}-\mathrm{C}_{3} \mathrm{~N}_{4} \\
(5 \mathrm{mg})\end{array}$ & $\begin{array}{l}\mathrm{Mo}-\mathrm{Mo}_{2} \mathrm{C} \\
(2.0 \mathrm{wt} \%)\end{array}$ & $\begin{array}{c}300 \mathrm{~W} \text { Xe lamp } \\
(\lambda>420 \mathrm{~nm})\end{array}$ & 219.7 & $\begin{array}{c}8.3 \% \\
(420 \mathrm{~nm})\end{array}$ & (7) \\
\hline $\begin{array}{c}\mathrm{Ni}_{2} \mathrm{P} / \mathrm{g}-\mathrm{C}_{3} \mathrm{~N}_{4} \\
(50 \mathrm{mg})\end{array}$ & $\begin{array}{c}\mathrm{Ni}_{2} \mathrm{P} \\
(3.5 \mathrm{wt} \%)\end{array}$ & $\begin{array}{c}300 \mathrm{~W} \text { Xe lamp } \\
(\lambda>420 \mathrm{~nm})\end{array}$ & 474.7 & $\begin{array}{c}3.2 \\
(435 \mathrm{~nm})\end{array}$ & (8) \\
\hline $\begin{array}{c}\mathrm{FeP} / \mathrm{g}-\mathrm{C}_{3} \mathrm{~N}_{4} \\
(60 \mathrm{mg})\end{array}$ & $\begin{array}{c}\mathrm{FeP} \\
(2.19 \mathrm{wt} \%)\end{array}$ & $\begin{array}{c}300 \mathrm{~W} \text { Xe lamp } \\
(\lambda>420 \mathrm{~nm})\end{array}$ & 177.9 & $\begin{array}{c}1.57 \\
(420 \mathrm{~nm})\end{array}$ & (9) \\
\hline $\begin{array}{c}\mathrm{CQD} / \mathrm{g}-\mathrm{C}_{3} \mathrm{~N}_{4} \\
(10 \mathrm{mg})\end{array}$ & $\begin{array}{c}\text { CQD } \\
(1 \mathrm{wt} \%)\end{array}$ & $\begin{array}{c}1000 \mathrm{~W} \text { Xe lamp } \\
(\lambda>420 \mathrm{~nm})\end{array}$ & 50.5 & $\begin{array}{c}1.4 \\
(405 \mathrm{~nm})\end{array}$ & (10) \\
\hline
\end{tabular}




\begin{tabular}{|c|c|c|c|c|c|}
\hline $\begin{array}{c}\mathrm{MoSe}_{2} / \mathrm{g}-\mathrm{C}_{3} \mathrm{~N}_{4} \\
(60 \mathrm{mg})\end{array}$ & $\begin{array}{c}\text { Network-like } \mathrm{MoSe}_{2} \\
(5 \mathrm{wt} \%)\end{array}$ & $\begin{array}{c}300 \mathrm{~W} \text { Xe lamp } \\
(\lambda>420 \mathrm{~nm})\end{array}$ & 136.8 & --- & (11) \\
\hline $\begin{array}{c}\mathrm{Ti}_{3} \mathrm{C}_{2} \mathrm{~T}_{\mathrm{X}} / \mathrm{g}-\mathrm{C}_{3} \mathrm{~N}_{4} \\
(3 \mathrm{mg})\end{array}$ & $\begin{array}{l}\mathrm{Ti}_{3} \mathrm{C}_{2} \mathrm{~T}_{\mathrm{X}} \\
(30 \mathrm{wt} \%)\end{array}$ & $\begin{array}{c}350 \mathrm{~W} \text { Xe lamp } \\
(\lambda>400 \mathrm{~nm})\end{array}$ & 88 & $\begin{array}{c}1.27 \\
\text { (unknown } \lambda \text { ) }\end{array}$ & (12) \\
\hline $\begin{array}{c}\mathrm{WC}_{1-\mathrm{X}} / \mathrm{g}-\mathrm{C}_{3} \mathrm{~N}_{4} \\
(50 \mathrm{mg})\end{array}$ & $\begin{array}{c}\mathrm{WC}_{1-\mathrm{x}} \\
(5 \mathrm{wt} \%)\end{array}$ & $\begin{array}{c}\text { solar simulator } \\
\text { AM } 1.5\end{array}$ & 2490 & $\begin{array}{c}4.38 \\
(400 \mathrm{~nm})\end{array}$ & $\begin{array}{c}\text { Our } \\
\text { Work }\end{array}$ \\
\hline
\end{tabular}

\section{References}

(1) Shao, M.; Shao, Y.; Ding, S.; Wang, J.; Xu, J.; Qu, Y.; Zhong, X.; Chen, X.; Ip, W. F.; Wang, N et al. Vanadium Disulfide Decorated Graphitic Carbon Nitride for Super-Efficient Solar-Driven Hydrogen Evolution. Appl. Catal. B Environ. 2018, 237, 295-301.

(2) Shao, M.; Shao, Y.; Chai, J.; Qu, Y.; Yang, M.; Wang, Z.; Yang, M.; Ip, W. F.; Kwok, C. T.; Shi, X et al. Synergistic Effect of $2 \mathrm{D} \mathrm{Ti}{ }_{2} \mathrm{C}$ and $\mathrm{g}_{-} \mathrm{C}_{3} \mathrm{~N}_{4}$ for Efficient Photocatalytic Hydrogen Production. J. Mater. Chem. A 2017, 5, 16748-16756.

(3) Akple, M. S.; Low, J.; Wageh, S.; Al-Ghamdi, A. A.; Yu, J.; Zhang, J. Enhanced Visible Light Photocatalytic $\mathrm{H}_{2}$-Production of $\mathrm{g}-\mathrm{C}_{3} \mathrm{~N}_{4} / \mathrm{WS}_{2}$ Composite Heterostructures. Appl. Surf. Sci. 2015, 358, 196-203.

(4) He, K.; Xie, J.; Yang, Z.; Shen, R.; Fang, Y.; Ma, S.; Chen, X.; Li, X. Earth-Abundant WC Nanoparticles as an Active Noble-Metal-Free Co-Catalyst for the Highly Boosted Photocatalytic $\mathrm{H}_{2}$ Production over g- $\mathrm{C}_{3} \mathrm{~N}_{4}$ Nanosheets under Visible Light. Catal. Sci. Technol. 2017, 7, 1193-1202.

(5) Song, Y.; Xia, K.; Gong, Y.; Chen, H.; Li, L.; Yi, J.; She, X.; Chen, Z.; Wu, J.; Li, H et al. Controllable Synthesized Heterostructure Photocatalyst $\mathrm{Mo}_{2} \mathrm{C} @ \mathrm{C} / 2 \mathrm{D} g-\mathrm{C}_{3} \mathrm{~N}_{4}$ : Enhanced Catalytic Performance for Hydrogen Production. Dalton Trans. 2018, 47, 14706-14712.

(6) He, K.; Xie, J.; Liu, Z.-Q.; Li, N.; Chen, X.; Hu, J.; Li, X. Multi-functional $\mathrm{Ni}_{3} \mathrm{C}$ Cocatalyst $/ \mathrm{g}_{-} \mathrm{C}_{3} \mathrm{~N}_{4}$ Nanoheterojunctions for Robust Photocatalytic $\mathrm{H}_{2}$ Evolution under Visible Light. J. Mater. Chem. A 2018, 6, 13110-13122.

(7) Dong, J.; Shi, Y.; Huang, C.; Wu, Q.; Zeng, T.; Yao, W. A New and Stable Mo-Mo ${ }_{2} \mathrm{C}$ Modified g-C $\mathrm{N}_{4}$ Photocatalyst for Efficient Visible Light Photocatalytic $\mathrm{H}_{2}$ Production. Appl. Catal. B Environ. 2019, 243, 27-35.

(8) Zeng, D.; Xu, W.; Ong, W.-J.; Xu, J.; Ren, H.; Chen, Y.; Zheng, H.; Peng, D.-L. Toward Noble-Metal-Free Visible-Light-Driven Photocatalytic Hydrogen Evolution: Monodisperse Sub-15nm Ni 2 P Nanoparticles Anchored on Porous g- $\mathrm{C}_{3} \mathrm{~N}_{4}$ Nanosheets to Engineer 0D-2D Heterojunction Interfaces. Appl. Catal. B Environ. 2018, 221, 47-55.

(9) Zeng, D.; Zhou, T.; Ong, W.-J.; Wu, M.; Duan, X.; Xu, W.; Chen, Y.; Zhu, Y.-A.; Peng, D.-L. Sub-5 nm Ultra-Fine FeP Nanodots as Efficient Co-Catalysts Modified Porous $\mathrm{g}_{-} \mathrm{C}_{3} \mathrm{~N}_{4}$ for Precious-Metal-Free Photocatalytic Hydrogen Evolution under Visible Light. ACS Appl. Mater. Interfaces 2019.

(10) Xia, X.; Deng, N.; Cui, G.; Xie, J.; Shi, X.; Zhao, Y.; Wang, Q.; Wang, W.; Tang, B. NIR Light Induced $\mathrm{H}_{2}$ Evolution by a Metal-Free Photocatalyst. Chem. Commun. 2015, 51, 10899-10902. 
(11) Zeng, D.; Wu, P.; Ong, W.-J.; Tang, B.; Wu, M.; Zheng, H.; Chen, Y.; Peng, D.-L. Construction of Network-Like and Flower-Like $2 \mathrm{H}-\mathrm{MoSe}_{2}$ Nanostructures Coupled with Porous $\mathrm{g}_{-} \mathrm{C}_{3} \mathrm{~N}_{4}$ for Noble-Metal-Free Photocatalytic $\mathrm{H}_{2}$ Evolution under Visible Light. Appl. Catal. B Environ. 2018, 233, 26-34.

(12) Sun, Y.; Jin, D.; Sun, Y.; Meng, X.; Gao, Y.; Dall'Agnese, Y.; Chen, G.; Wang, X.-F. g- $\mathrm{C}_{3} \mathrm{~N}_{4} / \mathrm{Ti}_{3} \mathrm{C}_{2} \mathrm{~T}_{\mathrm{x}}$ (MXenes) Composite with Oxidized Surface Groups for Efficient Photocatalytic Hydrogen Evolution. J. Mater. Chem. A 2018, 6, 9124-9131. 